\title{
Antioxidant status of surgical patients receiving TPN with an $\Omega$-3-fatty acid-containing lipid emulsion supplemented with $\alpha$-tocopherol
}

\author{
J. LINSEISEN* , J. HOFFMANN* , S. LIENHARD ${ }^{\dagger}$, K.-W. JAUCH ${ }^{\dagger}$, G. WOLFRAM* \\ ${ }^{*}$ Institute of Nutrition Science of the Technical University of Munich, Freising-Weihenstephan, ${ }^{\dagger}$ Department of \\ Surgery of the University of Regensburg, Regensburg, Germany (Correspondence to: JL Institut für \\ Ernährungswissenschaft der TU München, 85350 Freising-Weihenstephan, Germany)
}

Abstract-Background: LCT lipid emulsions and even more fish oil-containing lipid emulsions are under debate regarding their tocopherol and PUFA content as well as their effect on the antioxidative status especially in patients with oxidative stress.

Methods: Thirty-three patients undergoing major abdominal surgery were randomly assigned to receive either an $\alpha$-tocopherol-supplemented $(562 \mu \mathrm{mol} / \mathrm{l}) \mathrm{MCT} / \mathrm{LCT} / \mathrm{omega}-3$-acid triglycerides (MLF, 5/4/1 w/W/W, $20 \%$ ) emulsion or a soybean oil-based LCT emulsion (20\%). The TPN regimen continously provided $1.4 \mathrm{~g}$ fat $\mathrm{kg} \mathrm{bw}^{-1} \mathrm{~d}^{-1}$ over 5 days.

Results: Plasma antioxidant concentrations were strongly reduced by surgical treatment. Following 5 days of TPN with the MLF emulsion, mean plasma $\alpha$-tocopherol increased by $20.0 \mu \mathrm{mol} / \mathrm{l}(1.98 \mu \mathrm{mol} / \mathrm{mmol}$ lipid), while nearly no change was observed in the LCT emulsion group. In both groups, plasma concentrations of all non-supplemented antioxidants (vitamin C, carotenoids, selenium) as well as serum total antioxidant capacity further decreased during TPN. The concentrations of plasma cholesterol oxidation products as a measure of in vivo lipid peroxidation revealed no changes over the TPN period in either group.

Conclusion: In contrast to the LCT emulsion, administration of the $\alpha$-tocopherol supplemented MLF lipid emulsion normalized $\alpha$-tocopherol plasma concentrations. Despite its high long-chain PUFA content, no hint for increased lipid peroxidation was found.

Key words: Tocopherol; antioxidants; surgical patients; fish oil; cholesterol oxidation products, total parenteral nutrition

\section{Introduction}

For many years, parenteral administration of lipids aimed to provide the organism with an efficient fuel and to prevent or correct essential fatty acid deficiency (1). However, conventional soybean oil-based fat emulsions contain great amounts of linoleate (n-6) and insufficient amounts of antioxidants such as $\alpha$-tocopherol (2). They lead to an accumulation of linoleate in cell membrane phospholipids and are associated with an increased production of peroxidative catabolites. New preparations of lipid emulsions should be dedicated to better maintain membrane fatty acyl balance (n-6/n-3 ratio) and to stabilize or restore a suitable antioxidant status (2).

The ratio of n-6 and n-3 fatty acids in membrane phospholipids affects structural and functional properties of the cell membranes. Most importantly, n-3 fatty acids, namely eicosapentaenoic acid (EPA), compete with $\mathrm{n}-6$ arachidonic acid to produce other lines of eicosanoids that are less inflammatory and non-thrombogenic. The production and release of various cytokines, interleukines and interferons involved in immunoregulatory processes may be changed by n-3 PUFA administration, too. Hence, it seems reasonable to use fatty acids in order to modulate cell function and metabolism (1-6). Indeed, supplementation with n-3 fatty acids has been reported to improve various pathophysiological states such as cardiovascular disease, psoriasis or inflammatory bowel disease and is also expected to reveal favourable effects in postoperative state, after injury or during sepsis $(2,3)$. According to Tashiro et al. (7), administration of $n-3$ polyunsaturated fatty acids (PUFA) improved the stress response and modulated the immune status after surgery. In postoperative patients a fast enrichment of plasma and platelet phospholipids with EPA as well as effects on mediator generation in leucocytes and platelets were reported after intravenous administration of n-3 PUFA (8-10).

Fish oil was identified as a suitable source of very longchain n-3 PUFA. Since then, fish oil-containing lipid emulsions have proven to be well tolerated in humans, in terms of usual clinical and metabolic parameters (2, 7, 8, 11-13). However, information on the effects of fish oil-containing lipid emulsions on the (anti)oxidativ status is scarce. Due to the high degree of unsaturation EPA and DHA are much more susceptible to oxidation than linoleic acid. Therefore, fish oil-containing lipid emulsions are supplemented with antioxidants such as $\alpha$-tocopherol to counteract peroxida- 
tion reactions. Regarding LCT emulsions which are low in $\alpha$-tocopherol, their susceptibility to oxidation was shown during storage (14-16) as well as after their infusion as identified by an increased breath pentane production (17, 18 ) or a decreased number of enlarged LDL particles (19). Contrary to this, in rats receiving TPN with a fish oil emulsion containing very high amounts of tocopherol homologues, no hints for increased autoxidation reactions were noted (20).

Therefore, the aim of the present study was to evaluate the antioxidant status in surgical patients as affected by the intravenous administration of two lipid emulsions largely differing in their fatty acid pattern and $\alpha$-tocopherol content.

\section{Materials and methods}

\section{Patients}

Thirty-three adult patients with upper and lower gastrointestinal malignancies, adenomas $(n=2)$, or stenosis of the small intestine $(n=1)$ who were undergoing major abdominal surgery entered the study. Patients were eligible for study entry when achieving the following criteria: no pregnancy, no lipid metabolism disorder (serum triacylglycerol $<250 \mathrm{mg} / \mathrm{dl}$, serum total cholesterol $<300 \mathrm{mg} / \mathrm{dl}$ ), no severe hepatic dysfunction, no severe blood clotting disorders, no hemorrhagic necrotizing pancreatitis, no acute thromboembolic events, no severe septic conditions. Characteristics of the patients are given in Table 1.

The study was approved by the Ethics Committee of the Medical Faculty of the University of Regensburg, and the procedures followed were in accordance with the Helsinki Declaration of 1975, as revised in 1989. All patients gave informed consent before commencement of the investigation. The study was performed as a randomized, doubleblinded, and prospective trial between February and June 1997. Patients were randomly assigned to two groups receiving an identical TPN regimen differing only in the fat component.

\section{TPN regimen}

The nutrition regimen consisted of $1.33 \mathrm{~g}$ amino acids $\mathrm{kg}$ body weight (bw) $)^{-1} \mathrm{~d}^{-1}, 4.2 \mathrm{~g}$ glucose $\mathrm{kg} \mathrm{bw}^{-1} \mathrm{~d}^{-1}$, and $1.4 \mathrm{~g}$ fat $\mathrm{kg} \mathrm{bw}^{-1} \mathrm{~d}^{-1}$, alltogether providing $35 \mathrm{kcal} \mathrm{kg} \mathrm{bw}^{-1} \mathrm{~d}^{-1}$. As

Table 1 Characteristics of the patients

\begin{tabular}{lll}
\hline & \multicolumn{2}{c}{ Group } \\
& MLF & LCT \\
\hline No. of patients & 17 & 16 \\
Mean age (y) & $60.7 \pm 8.7$ & $59.4 \pm 12.6$ \\
Sex (M/F) & $10 / 7$ & $13 / 3$ \\
Body height (m) & $1.70 \pm 0.09$ & $1.71 \pm 0.09$ \\
Body weight $(\mathrm{kg})$ & $74.9 \pm 7.2$ & $79.0 \pm 13.8$ \\
Body mass index $\left(\mathrm{kg} / \mathrm{m}^{2}\right)$ & $25.9 \pm 2.5$ & $27.0 \pm 4.9$ \\
Operation procedures $(\mathrm{n})$ & & 5 \\
$\quad$ Gastrectomy & 4 & 10 \\
$\quad$ Resection of colon, rectum & 11 & 1 \\
$\quad$ Miscellaneous & 2 & \\
\hline
\end{tabular}

a lipid component either a soybean oil emulsion (LCT; Intralipid $^{\circledR} 20 \%$; Pharmacia GmbH, Erlangen Germany) or an omega-3-fatty acid-containing fat emulsion (MLF, Lipoplus ${ }^{\circledR}$ 20\%; B. Braun Melsungen AG, Melsungen, Germany) were used. Fatty acid composition and antioxidant content are given in Table 2. Fat emulsion $(0.51)$ was added to 21 Nutriflex ${ }^{\circledR}$ plus (B. Braun Melsungen) to give an all-in-one mixture. The mixture also provided sodium, potassium, calcium, magnesium, chloride, and phosphate. Additionally, trace elements and vitamins were administered. On the first and second postoperative day, the TPN dose was restricted to $50 \%$. The TPN was delivered continuously over $22-24 \mathrm{~h}$ per day on 5 consecutive days, beginning at the 1 st postoperative day in the morning (day 1) and ending at the 6th postoperative day in the morning (day 6).

\section{Blood sampling}

Blood samples were drawn before surgery (day 0, fasted), on the 1st postoperative day in the morning before starting infusion (day 1, fasted), and on the 6th postoperative day in the morning before the infusion was stopped (day 6, end of infusion). Tubes containing the antioxidants EDTA $(1.6 \mathrm{mg} / \mathrm{ml}$ blood $)$ and TROLOX $(1 \mu \mathrm{mol} / \mathrm{l}$ blood; both from Sigma, Deisenhofen, Germany) were used for blood sampling. After plasma preparation the samples were stored at $-85^{\circ} \mathrm{C}$ until analysis.

\section{Plasma lipids and plasma phospholipid fatty acid profile}

Plasma total cholesterol, triglyceride and phospholipid concentrations were determined enzymatically using test com-

Table 2 Ingredients, fatty acid composition, and antioxidant content of the omega-3-fatty acid-containing lipid emulsion $\left(\mathrm{MLF}^{\$}\right)$ and the $\mathrm{LCT}^{\#}$ lipid emulsion

\begin{tabular}{|c|c|c|c|c|}
\hline & \multicolumn{4}{|c|}{ Lipid emulsion } \\
\hline & \multicolumn{2}{|c|}{$\mathrm{MLF}^{\$}$} & \multicolumn{2}{|c|}{$\mathrm{LCT}^{\#}$} \\
\hline & \multicolumn{2}{|c|}{ (wt.\%) } & \multicolumn{2}{|c|}{ (wt.\%) } \\
\hline MCT & \multicolumn{2}{|c|}{10.0} & \multicolumn{2}{|c|}{-} \\
\hline Soybean oil & \multicolumn{2}{|c|}{8.0} & \multicolumn{2}{|c|}{20.0} \\
\hline Omega-3-acid trigly & \multicolumn{2}{|c|}{2.0} & \multicolumn{2}{|c|}{-} \\
\hline Phospholipids (egg & \multirow{2}{*}{\multicolumn{2}{|c|}{1.2}} & \multicolumn{2}{|c|}{1.2} \\
\hline Glycerol & & & \multicolumn{2}{|c|}{2.25} \\
\hline Fatty acid & (wt.\%) & $(\mathrm{mol}-\%)$ & (wt.\%) & $(\mathrm{mol}-\%)$ \\
\hline $8: 0$ & 30.1 & 40.7 & - & - \\
\hline $10: 0$ & 19.4 & 22.2 & - & - \\
\hline $14: 0$ & 0.5 & 0.4 & 0.2 & 0.3 \\
\hline $16: 0$ & 5.9 & 4.7 & 10.5 & 11.2 \\
\hline $16: 1$ & 0.6 & 0.5 & 0.1 & 0.1 \\
\hline $18: 0$ & 2.4 & 1.7 & 3.7 & 3.6 \\
\hline $18: 1$ & 7.9 & 5.7 & 20.9 & 20.3 \\
\hline $18: 2 n-6$ & 24.4 & 17.7 & 56.6 & 55.2 \\
\hline $18: 3 n-3$ & 3.3 & 2.4 & 7.8 & 7.7 \\
\hline $20: 5 n-3$ & 3.1 & 2.1 & - & - \\
\hline $22: 6 n-3$ & 2.3 & 1.4 & - & - \\
\hline Antioxidant & $(\mathrm{mg} / \mathrm{l})$ & $(\mu \mathrm{gmol} / 1)$ & $(\mathrm{mg} / \mathrm{l})$ & $(\mu \mathrm{gmol} / 1)$ \\
\hline$\alpha$-Tocopherol & $242.2^{\sim}$ & $562.3^{\sim}$ & 22.0 & 51.1 \\
\hline$\gamma$-Tocopherol & 100.8 & 241.9 & 143.6 & 344.6 \\
\hline Ascorbyl palmitate & $115.2^{\sim}$ & $277.9^{\sim}$ & - & - \\
\hline
\end{tabular}

${ }^{\$}$ MLF emulsion: Lipoplus ${ }^{\circledR}$; ${ }^{\#}$ LCT emulsion: Intralipid ${ }^{\circledR} ; ~ \sim$ Supplemented by the manufacturer of the lipid emulsion, including naturally occurring $\alpha$-tocopherol from soybean oil (range of $20-40 \mathrm{mg} / \mathrm{l}$ ). 
binations ('Monotest Cholesterin', 'Triglyceride GPO-PAP', 'Test-Combination Phospholipide'; all Boehringer Mannheim GmbH, Mannheim, Germany).

After solvent extraction (21), the plasma lipids were separated by means of thin layer chromatography (silica plates) using petrol ether/ethylmethylketone/acetic acid $=84 / 15 / 1$ $(\mathrm{v} / \mathrm{v} / \mathrm{v})$ as solvent agent. Fatty acid methyl ester (FAME) of phospholipids were obtained by transesterification with TMSH (22). The FAME were separated by means of a CPSil-88 capillary column (Chrompack, Frankfurt, Germany), installed in an HP 5890 gas chromatograph with FID (Hewlett Packard, Taufkirchen, Germany). For identification and quantification of the FAME peaks, standard reagents of analytical grade were used.

\section{Determination of tocopherols, carotenoids, vitamin $C$, selenium, and antioxidant capacity}

Aliquots of the plasma samples before and after LDLapheresis were analysed for $\alpha$-tocopherol and carotenoid concentrations by HPLC according to the described method (23). Calculations were made using the internal standard method and relative response factors. As internal standards, $\beta$-apo- 8 -carotenoic acid ethyl ester and DL- $\alpha$-tocopheryl acetate were added. Recovery of the substances after enrichment of serum samples was within 94 and $107 \%$ with coefficients of variation $<2.3 \%(n=5)$.

Vitamin C content of plasma samples was analysed photometrically $(520 \mathrm{~nm})$ according to Speitling et al. (24). Ascorbic acid (Sigma, Deisenhofen, Germany) standard solution was taken for calibration and quality control. Mean recovery of ascorbic acid added to plasma samples $(n=7)$ was $101.6 \%$ with a coefficient of variation of $0.7 \%$.

Plasma selenium concentrations were determined by graphite furnance atomic absorption spectrometry, using a Perkin-Elmer 2380 atomic absorption spectrophotometer, a HGA 500 graphite furnance as well as pyrolytically coated graphite tubes with an inserted L'vov platform and a hallowcathode lamp for selenium (Perkin-Elmer, Überlingen, Germany). Sample preparation and analysis conditions were adapted from Welz et al. (25). Quality control was carried out using Seronorm Trace Elements Serum (Promochem, Wesel, Germany). Mean recovery of selenium (standard solution) added to plasma samples $(n=4)$ was $96.9 \%$ with a coefficient of variation of $6.7 \%$.

Total antioxidant capacity of the serum samples was measured with a chemiluminescent assay (26) using horseradish peroxidase (E.C. 1.11.1.7; Boehringer Mannheim $\mathrm{GmbH}$ ) and ELC immunoassay signal reagent (Amersham International plc, Buckinghamshire, UK), providing luminol, p-iodophenol (enhancer) and hydrogen peroxide (oxidant). Measurement of light emission was performed by means of a Biolumat LB 9500 T (Laboratorium, Professor Dr Berthold, Wildbad, Germany). Calibration was carried out within each series using TROLOX (Sigma) standard solutions and antioxidant capacity was expressed in $\mu \mathrm{mol}$ TROLOX equivalent/l serum. Mean recovery of TROLOX added to serum samples $(n=7)$ was $102 \%$ with a coefficient of variation of $2.2 \%$.

\section{Analysis of cholesterol oxidation products (COP)}

The plasma samples were analysed for the COP content in their total cholesterol fraction. Cholesteryl esters were enzymatically hydrolyzed by cholesterol esterase (E.C. 1.1.1.13, Sigma). The further methodology is described elsewhere in detail (27). 5-Pregnen-3 $\beta$-ol-7,20-dione (7-ketopregnenolone) was used as an internal standard. All standard substances were delivered by Steraloids Ltd (New Barnett, UK) and Sigma. Identification of the sample peaks was made by retention times of the standard substances and the characteristic ion fragmentation. For quantification a selected ion monitoring program was used. Recovery of free COP standard substances was in the range of 85 (cholestanetriol) $123 \%$ (25-hydroxycholesterol); mean recovery for the sum of COP was $99 \%$. Mean coefficient of variation for the determination of COP in plasma was $2.3 \%$ with a range of 1.9 (7 $\alpha$-hydroxycholesterol) $-5.3 \%$ (25-hydroxycholesterol).

\section{Statistics}

The results are given in terms of mean and standard error of mean (SEM). Statistical analysis was performed with SPSS, Version 7.5 (SPSS Inc., Chicago, USA). For analysis of variance a two-factorial model with the factors 'fat emulsion' and 'time' was applied; comparisons of means were made with the unpaired $t$-test at an $\alpha$-level of $5 \%$.

\section{Results}

All patients enrolled completed the investigation; no adverse drug reactions were noted. After 5 days of TPN with the omega-3-fatty acid-containing fat emulsion, plasma phospholipids contained a significantly higher portion of eicosapentaenoic acid (2.07 mol-\%) than found after administration of the soybean fat emulsion ( 0.77 mol-\%; Table 3$)$. The mean difference in docosahexaenoic acid (DHA) concentration between groups was not of statistical significance. The mean portion of linoleic acid in plasma phospholipids was significantly higher after administration of the LCT emulsion compared to the MLF emulsion.

The plasma concentrations of selected antioxidants and the total antioxidant capacity (TAC) are given in Table 4 . Statistically significant differences between groups were found for $\alpha$-tocopherol and $\gamma$-tocopherol. Due to differences in the mean carotenoid concentrations on day 0 and 1 between the two groups, the change during the infusion period ( $\Delta$ day 6-day 1) was calculated. However, these values do not indicate significantly different effects of the two fat emulsions on carotenoids, vitamin $\mathrm{C}$ or selenium (Table 4). Moreover, after correction for plasma lipids, only the changes in plasma $\alpha$ - and $\gamma$-tocopherol were found to be significantly affected by the type of fat emulsion administered (Table 5) but in both groups the decrease of TAC values could not be stopped. 
Table 3 Fatty acid composition (mol-\%, mean \pm SEM) of plasma phospholipids in surgical patients before (day 0, fasted) and after surgery (day 1 , fasted) as well as after 5 days of TPN (day 6, end of infusion) with an omega-3-fatty acid-containing lipid emulsion (MLF, $n=17$ ) or an LCT emulsion ( $n=16$ )

\begin{tabular}{|c|c|c|c|c|c|c|}
\hline \multirow[b]{2}{*}{ Fatty acid } & \multicolumn{2}{|c|}{ Day 0} & \multicolumn{2}{|c|}{ Day 1} & \multicolumn{2}{|c|}{ Day 6} \\
\hline & MLF & LCT & MLF & LCT & MLF & LCT \\
\hline $16: 0$ & $34.80 \pm 0.71$ & $34.78 \pm 0.80$ & $37.59 \pm 0.56$ & $36.66 \pm 0.98$ & $33.69 \pm 1.10$ & $31.75 \pm 1.07$ \\
\hline 18:0 & $15.34 \pm 0.52$ & $15.72 \pm 0.47$ & $14.54 \pm 0.57$ & $14.38 \pm 0.49$ & $18.08 \pm 0.63$ & $16.22 \pm 0.51$ \\
\hline $18: 2 n-6$ & $17.30 \pm 0.89$ & $16.97 \pm 0.73$ & $16.39 \pm 0.73$ & $15.88 \pm 0.79$ & $14.99 \pm 0.71 *$ & $20.08 \pm 1.01 *$ \\
\hline $18: 3 n-3$ & $0.51 \pm 0.21$ & $0.45 \pm 0.25$ & $0.23 \pm 0.04$ & $0.34 \pm 0.13$ & $0.42 \pm 0.08$ & $1.12 \pm 0.56$ \\
\hline $20: 3 n-6$ & $1.97 \pm 0.14$ & $2.22 \pm 0.15$ & $1.69 \pm 0.12$ & $2.03 \pm 0.20$ & $1.78 \pm 0.19$ & $2.04 \pm 0.23$ \\
\hline $22: 6 n-3$ & $5.29 \pm 0.60$ & $5.01 \pm 0.46$ & $5.05 \pm 0.48$ & $5.67 \pm 0.66$ & $5.68 \pm 0.98$ & $5.72 \pm 0.67$ \\
\hline$\sum n-6^{\#}$ & $28.03 \pm 1.18$ & $27.50 \pm 1.13$ & $27.03 \pm 0.74$ & $26.52 \pm 1.18$ & $22.99 \pm 1.06$ & $28.25 \pm 1.70$ \\
\hline $\bar{\sum} n-3^{\S}$ & $6.37 \pm 0.76$ & $6.32 \pm 0.70$ & $5.93 \pm 0.50$ & $6.68 \pm 0.73$ & $8.17 \pm 1.09$ & $7.61 \pm 1.26$ \\
\hline$\sum$ n-6:n-3 ratio & $5.27 \pm 0.55$ & $5.14 \pm 0.58$ & $5.12 \pm 0.50$ & $4.82 \pm 0.70$ & $3.66 \pm 0.47$ & $4.38 \pm 0.38$ \\
\hline
\end{tabular}

${ }^{\#} 18: 2 n-6+20: 3 \mathrm{n}-6+20: 4 \mathrm{n}-6 ;{ }^{\S} 18: 3 \mathrm{n}-3+20: 5 \mathrm{n}-3+22: 6 \mathrm{n}-3$; * significantly different means by treatment, $P \leq 0.05$; unpaired $t$-test.

Table 4 Antioxidant concentrations (mean \pm SEM, $\mu$ mol/l) and total antioxidative capacity (TAC, $\mu$ mol TROLOX-eq./l) in the plasma of surgical patients before (day 0 , fasted) and after surgery (day 1 , fasted) as well as after 5 days of TPN (day 6 , end of infusion) with an omega-3-fatty acid-containing lipid emulsion (MLF; $n=17$ ) or an LCT emulsion $(n=16)$

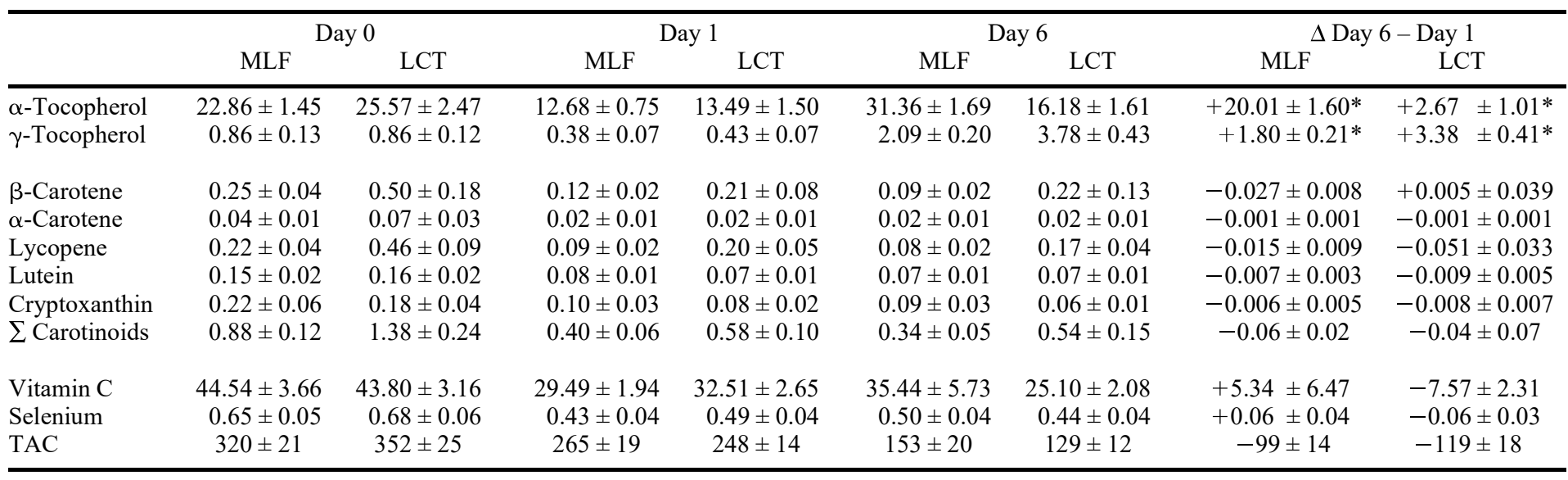

*Significantly different means by treatment; $P<0.01$; unpaired $t$-test.

Table 5 Plasma lipids and lipid-related antioxidant concentrations (mean \pm SEM; $\mu$ mol, nmol antioxidant/ $\mu \mathrm{mmol}$ plasma lipids ${ }^{\#}$ ) in the plasma of surgical patients before (day 0 , fasted) and after surgery (day 1 , fasted) as well as after 5 days of TPN (day 6 , end of infusion) with an omega-3-fatty acid-containing lipid emulsion (MLF; $n=17)$ or an LCT emulsion $(n=16)$

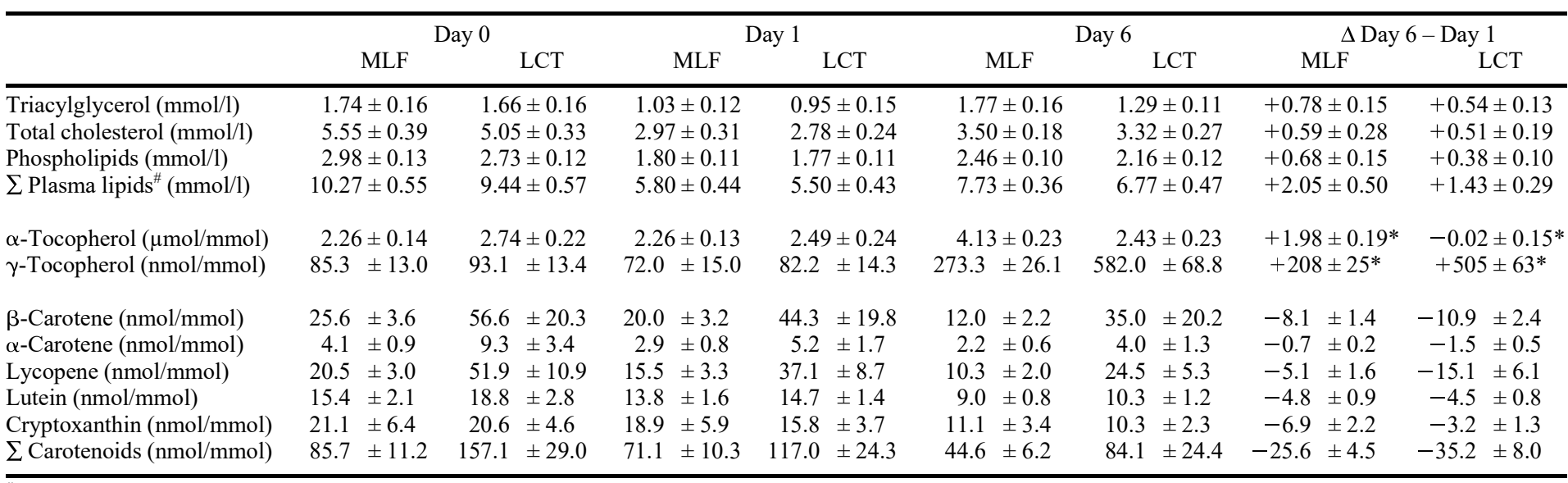

${ }^{\#}$ Sum of plasma triacylglycerol, total cholesterol, and phospholipids; *significantly different means by treatment; $P<0.001$, unpaired $t$-test. 
Table 6 Cholesterol oxidations products (COP; $\mu \mathrm{mol} / 1$, mean $\pm \mathrm{SEM})$, total cholesterol $(\mu \mathrm{mmol} / \mathrm{l})$ and COP:cholesterol ratio $(\mathrm{mol} / \mathrm{mmol})$ in the plasma of surgical patients before (day 0 , fasted) and after surgery (day 1, fasted) as well as after 5 days of TPN (day 6, end of infusion) with an omega-3-fatty acid-containing lipid emulsion (MLF; $n=9$ ) or an LCT emulsion $(n=6)$

\begin{tabular}{|c|c|c|c|c|c|c|}
\hline & \multicolumn{2}{|c|}{ Day 0} & \multicolumn{2}{|c|}{ Day 1} & \multicolumn{2}{|c|}{ Day 6} \\
\hline & MLF & LCT & MLF & LCT & MLF & LCT \\
\hline $7 \alpha$-Hydroxycholesterol $(\mu \mathrm{mol} / \mathrm{l})$ & $12.50 \pm 1.34$ & $10.33 \pm 0.58$ & $12.07 \pm 1.05$ & $10.46 \pm 0.50$ & $11.35 \pm 1.21$ & $10.32 \pm 0.31$ \\
\hline $7 \beta$-Hydroxycholesterol $(\mu \mathrm{mol} / \mathrm{l})$ & $13.51 \pm 1.33$ & $11.44 \pm 0.58$ & $13.26 \pm 0.99$ & $11.52 \pm 0.56$ & $12.56 \pm 1.26$ & $11.55 \pm 0.27$ \\
\hline Cholesterol- $\alpha$-epoxide $(\mu \mathrm{mol} / \mathrm{l})$ & $1.59 \pm 0.32$ & $0.95 \pm 0.13$ & $1.27 \pm 0.14$ & $1.07 \pm 0.06$ & $1.58 \pm 0.30$ & $1.02 \pm 0.12$ \\
\hline Cholestanetriol $(\mu \mathrm{mol} / \mathrm{l})$ & $0.52 \pm 0.10$ & $0.45 \pm 0.08$ & $0.38 \pm 0.08$ & $0.50 \pm 0.12$ & $0.62 \pm 0.10$ & $0.41 \pm 0.09$ \\
\hline 6-Ketocholestanol $(\mu \mathrm{mol} / \mathrm{l})$ & $0.04 \pm 0.02$ & $0.04 \pm 0.02$ & $0.05 \pm 0.02$ & $0.03 \pm 0.02$ & $0.31 \pm 0.16$ & $0.03 \pm 0.02$ \\
\hline$\sum \mathrm{COP}(\mu \mathrm{mol} / \mathrm{l})$ & $45.93 \pm 3.71$ & $39.3 \pm 22.71$ & $45.90 \pm 3.44$ & $40.26 \pm 2.00$ & $45.50 \pm 4.22$ & $40.86 \pm 1.32$ \\
\hline Total cholesterol (mmol/l) & $5.55 \pm 0.39$ & $5.05 \pm 0.33$ & $2.97 \pm 0.31$ & $2.78 \pm 0.24$ & $3.50 \pm 0.18$ & $3.32 \pm 0.27$ \\
\hline COP:cholesterol ratio $(\mu \mathrm{mol} / \mathrm{mmol})$ & $8.18 \pm 0.86$ & $8.48 \pm 0.94$ & $18.43 \pm 2.18$ & $15.40 \pm 1.40$ & $13.45 \pm 1.58$ & $12.20 \pm 0.62$ \\
\hline
\end{tabular}

As a measure of in vivo oxidation processes, plasma total COP concentrations were analysed. The results show only small changes by time (Table 6). The insignificant differences between groups existed on all blood sampling points. Due to lower plasma total cholesterol concentrations on days 1 and 6 (versus day 0), the COP: cholesterol ratio increased to a similar extent in both groups.

\section{Discussion}

The plasma lipid concentrations were not significantly different between groups and confirm an effective hydolysis and plasma clearance of the administered MLF emulsion (2). Plasma phospholipids were significantly enriched with EPA after 5 days of TPN with the MLF fat emulsion $(+1.62$ mol- $\%$, day 6 vs day 1$)$; the mean increase in DHA amounting to +0.63 mol- $\%$ did not reach statistical significance. Using a comparable study design, Morlion and coworker (8) reported a similar enrichment of EPA in plasma phospholipids after 5 days of infusion of a fish oil-containing fat emulsion. The contribution of soybean oil to the administered MLF fat emulsion amounted to $40 \%$ of the total fat content; consequently, the linoleic acid content (linoleic acid as the most important fatty acid of soybean oil) was approximately $60 \%$ lower compared to the LCT emulsion (Table 2). As expected, LCT infusion provoked significantly higher linoleic acid concentrations in plasma phospholipids than MLF infusion (Table 3). For the differences in the calculated sums of the n-3 and n- 6 fatty acids no statistical significance between groups could be obtained. Thus, the lower n-6:n-3 ratio in the MLF group after 5 days of TPN was not significantly different from that found for the LCT group. However, Lienhard et al. (28) demonstrated, for the patients of the present investigation, that the infused amount of $n-3$ fatty acids was sufficient to reveal biological effects.

Patients with major surgery undergo a special metabolic stress situation that is coupled with an increased production of radicals and other highly reactive compounds mainly originating from immunologically active cells $(29,30)$. In particular, in dealing with the problem of ischemia reperfusion injury supplementation with antioxidants such as vitamin $\mathrm{E}$ was shown to reduce the production of free radicals and lipid peroxidation products and to decrease cell damage, indicating a clear benefit for the clinical outcome of the patients (30-33). It can be deduced that the preoperative antioxidant status is as important as its development after surgery. Moreover, intravenous administration of lipid emulsions containing a high amount of PUFA and an insufficient protection by antioxidants may provoke undesirable autoxidation processes (2). The susceptability of fatty acids for oxidation strongly increases with the number of double bonds. Hence, the highly unsaturated fatty acids EPA and DHA from fish oil reveal a high risk for oxidative damage and deserves a careful handling (e.g. light protection) and a sufficient content of antioxidative substances as well.

Dupont et al. (15) demonstrated that a similar fish oilcontaining fat emulsion (MLF 541, $200 \mathrm{mg} / \mathrm{l} \alpha$-tocopherol) was efficiently resistent to in vitro oxidation. They found comparable results for the oxidative stability with the MLF emulsion and the MCT/LCT (1/1) emulsion, while the soybean oil fat emulsion (LCT) with low $\alpha$-tocopherol content $(15 \mathrm{mg} / \mathrm{l})$ was shown to be much more sensitive to in vitro peroxidation. The authors concluded that the oxidative risk by fish oil is efficiently counteracted by the $\alpha$-tocopherol enrichment (15). However, at rather high concentrations the antioxidant activity of $\alpha$-tocopherol can be reversed to a prooxidant effect in certain in vitro models $(34,35)$, indicating an upper limit for the $\alpha$-tocopherol supplementation of lipid emulsions. According to the findings of Dupont (16), this limit may not be exceeded by addition of $200 \mathrm{mg} / 1$ $\alpha$-tocopherol.

Regarding the essentiality of vitamin E, $\gamma$-tocopherol shows a much lower biological acitivity than $\alpha$-tocopherol after dietary intake (based on bioassays in animals) (36) but reveals the highest antioxidant efficacy among the tocopherol homologues when tested in foodstuff (37). However, Traber et al. (38) showed that after stopping the 
infusion of soybean oil-based lipid emulsions the elevated plasma $\gamma$-tocopherol levels could not be maintained. They supposed that $\gamma$-tocopherol is rapidly taken up by the liver and not further released via VLDL secretion.

They also found that the TG emulsion particles enrich $\alpha$-tocopherol by depleting endogenous lipoproteins (LDL). Further, the elevated pentane exhalation in patients on total parenteral nutrition with soybean oil-based lipid emulsions hints to an incomplete protection of PUFA by $\gamma$-tocopherol $(17,18)$. It seems likely that even high amounts of non- $\alpha-$ tocopherol do not equal $\alpha$-tocopherol in parenteral nutrition (38-40). Finally, less is known about the biological consequences of a non- $\alpha$-tocopherol enrichment of cell membranes (40). Only after a sufficient supplementation of $\alpha$-tocopherol, also considering the ratio of $\alpha$-tocopherol to non- $\alpha$-tocopherol, plasma levels of $\alpha$-tocopherol could be distinctly enhanced and maintained after stopping the infusion (38).

Intravenous provision of the recommended dietary allowance (RDA) of $\alpha$-tocopherol was already shown to be unable to stop the decrease in plasma or red blood cell $\alpha$-tocopherol concentrations in different patients receiving $\operatorname{TPN}(39,40)$. For the purpose of parenteral nutrition a sufficient intake of vitamin $\mathrm{E}$ and other antioxidants is better judged on their plasma levels. Regarding the results of prospective studies on the preventive effect of antioxidants, i.e., a low risk for coronary heart disease and certain types of cancer, $\alpha$-tocopherol, vitamin $C$, and $\beta$-carotene plasma concentrations of $>30 \mu \mathrm{mol} / 1,>50 \mu \mathrm{mol} / 1$, and $>0.4 \mu \mathrm{mol} / 1$ respectively were regarded as optimum levels (41). In the VERA study (Germany), low plasma concentrations were identified by means of the 2.5 th percentiles of a reference sample with values $<17.7 \mu \mathrm{mol} / 1$ for $\alpha$-tocopherol, $<36.8 \mu \mathrm{mol} / 1$ for vita$\min C$, and $<0.18 \mu \mathrm{mol} / 1$ for $\beta$-carotene (42). Evaluating the plasma antioxidant concentrations before surgery (day 0 ; Table 4), the average of the patients laid below the given optimum values, but nearly all patients exceeded the 2.5 th percentiles. After surgery (day 1), the concentrations of all antioxidants were drastically reduced. Due to the concomittantly reduced plasma lipid concentrations the decreases were much smaller or even disappeared when the lipid-soluble antioxidants were corrected for the plasma lipid content (Table 5); this is especially true for $\alpha$-tocopherol.

During the 5 day TPN period, $\alpha$ - and $\gamma$-tocopherol concentrations (in $\mu \mathrm{mol} / 1$, Table 4 ) increased in both TPN treatment groups. The changes in plasma concentrations were in accordance to the proportion of the tocopherol homologues in the two fat emulsions.

However, after correction for plasma lipids the $\alpha$-tocopherol values in the LCT group did not change at all during TPN (Table 5), indicating that the (-tocopherol content of the LCT emulsion was insufficient to reconstitute preoperative plasma values or to reach recommended plasma concentrations (see above). In contrast, infusion of the MLF emulsion resulted in desirable plasma $\alpha$-tocopherol concentrations. A supplementation dose of $200 \mathrm{mg} \alpha$-tocopherol per day was already reported to normalize plasma $\alpha$-tocopherol values in patients on long-term home parenteral nutrition (19).
On average, the patients' initial plasma concentrations of $\alpha$-carotene, lycopene, lutein, and cryptoxanthin were rather low, judged by means of reported 'typical' plasma concentrations (43). This is also true for selenium (44). Due to the ascorbyl palmitate supplementation of the MLF emulsion, the fall in plasma vitamin $\mathrm{C}$ concentrations by surgical treatment could be stopped in the patients of the MLF group only. Contrary to this, the plasma carotenoid concentrations continously decreased in both groups, caused by the lack of intake during the observational period and the metabolic stress situation. It is worth mentioning that carotenoids - as well as tocopherols - may not only be involved in the management of oxidative stress but may also reveal effects on the immune system at a cellular level $(36,45)$.

Surprisingly, the high plasma $\alpha$-tocopherol concentrations in the MLF group on day 6 were not accompanied by enhanced TAC values (Table 4). However, it has to be stressed that the TAC is strongly affected by other plasma antioxidants of endogene origin, i.e., uric acid, bilirubin, and protein sulfhydryl groups, not determined here $(26,46)$. TAC values before surgery were at the lower physiological range of $414.7 \pm 77.3 \mu \mathrm{mol} / 1(26)$.

As suggested by Jialal et al. (47), measurement of COP in plasma or lipoproteins may serve as an index of in vivo oxidation reactions. The results provide better information than given by in vitro assays, e.g. the determination of the oxidative susceptability of LDL in response to the addition of an oxidant or the determination of the total antioxidant capacity in serum samples. The reported plasma COP concentrations gave no distinct hint for enhanced in vivo oxidation processes after administration of the MLF fat emulsion. The COP concentrations were rather stable throughout the study (Table 6). Not even the surgical treatment seemed to be followed by increased plasma cholesterol oxidation (day 1 vs day 0). However, plasma concentrations may not reflect the situation in target tissues after surgery. Besides, it should be mentioned that, postoperatively, plasma cholesterol concentrations decreased, resulting in higher COP:cholesterol ratios. Since it is suspected that only highly COP-enriched lipoprotein (LDL) particles are atherogenic (48), the situation may have been worsened by surgery. Compared to healthy young subjects revealing mean plasma COP concentrations of $31.2 \pm 0.6 \mu \mathrm{mol} / 1$ (49), the patients had elevated plasma COP values before surgical treatment. However, values of a well-matched control group are not available.

In conclusion, the $\alpha$-tocopherol enrichment of the omega3-fatty acid-containing fat emulsion was able to normalize low plasma values in patients after surgery. Without supplementation, plasma concentrations of all carotenoids, vitamin $C$, and selenium decreased by surgery and the fall continued over the following 5 days of TPN. However, the total plasma COP concentrations as a measure of autoxidation processes in vivo were neither affected by surgery nor by TPN. While the need for $\alpha$-tocopherol enrichment of lipid emulsions seems generally accepted, the supplementation of other antioxidants within TPN needs further evaluation since the decrease in serum total antioxidant capacity could not be stopped by $\alpha$-tocopherol supplementation only. 


\section{References}

1. Wolfram G. The use of lipid infusions in postoperative nutrition. Nutrition 1998; 14: 407-409

2. Carpentier Y A, Simoens C, Siderova V, El Nakadi I, Vanweyenberg V, Eggerickx D, Deckelbaum R J. Recent developments in lipid emulsions: relevance to intensive care. Nutrition 1997; 13: 73S-78S

3. Fürst P. New parenteral substrates in clinical nutrition. Part II. New substrates in lipid nutrition. Eur J Clin Nutr 1994; 48: 681-691

4. Grimm H, Tibell A, Norrlind B, Blecher C, Wilker S, Schwemmle K Immunoregulation by parenteral lipids: impact of the $n-3$ to $n-6$ fatty acid ratio. JPEN 1994; 18: 417-421

5. Grimble R F, Tappia P S. Modulation of pro-inflammatory cytokine biology by unsaturated fatty acids. Z Ernährungswiss 1998; 37: $57-65$

6. Kinsella J E, Lokesh B, Broughton S, Whelan J. Dietary polyunsaturated fatty acids and eicosanoids: potential effects on the modulation of inflammatory and immune cells: an overview. Nutrition 1990; 6: 24-44

7. Tashiro T, Yamamori H, Takagi K, Hayashi N, Furukawa K, Nakyjima N. n-3 verus n-6 polyunsaturated fatty acids in critical illness. Nutrition 1998; 14: 551-553

8. Morlion B J, Torwesten E, Lessire H, Sturm G, Peskar B M, Fürst P, Puchstein C. The effect of parenteral fish oil on leucocyte membrane fatty acid composition and leucotriene-synthesizing capacity in patients with postoperative trauma. Metabolism 1996; 45: $1208-1213$

9. Wachtler P, König W, Senkal M, Kemen M, Koller M. Influence of a total parenteral nutrition enriched with omega-3 fatty acids on leukotriene synthesis of peripheral leukozytes and systemic cytokine levels in patients with major surgery. J Trauma 1997; 42: 191-198

10. Roulet M, Frascarolo P, Pilet M, Chapuis G. Effects of intravenously infused fish oil on platelet fatty acid phospholipid composition and on platelet function in postoperative trauma. JPEN 1997; 21 : 296-301

11. Katz D P, Manner T, Furst P, Askanazi J. The use of an intravenous fish oil emulsion enriched with omega-3 fatty acids in patients with cystic fibrosis. Nutrition 1996; 12: 334-339

12. Elmadfa I, Stroh S, Brandt K, Schlotzer E. Influence of a single parenteral application of a $10 \%$ fish oil emulsion on plasma fatty acid pattern and the function of thrombocytes in young adult men. Ann Nutr Metab 1993; 37: 8-13

13. Oliveira F L, Rumsey S C, Schlotzer E, Hansen I, Carpentier Y A, Deckelbaum R J. Triglyceride hydrolysis of soy oil vs fish oil emulsions. JPEN 1997; 21: 224-229

14. Pitkänen O, Hallman M, Andersson M. Generation of free radicals in lipid emulsions used in parenteral nutrition. Pediatr Res 1991; 29: 56-59

15. Dupont I, Deckelbaum R J, Carpentier Y A. Influence of triglyceride (TG) fatty acid pattern and $\alpha$-tocopherol $(\alpha$-toc) content on the sensitivity of i.v. lipid emulsions to peroxidative damage. Clin Nutr 1998; 17: 59

16. Dupont I E. Peroxidation of lipid emulsions: effects of changes in fatty acid pattern and $\alpha$-tocopherol content on the sensitivity to peroxidative damage. Clin Nutr 1999; 18: 113-116

17. Van Gossum A, Shariff R, Lemoyne M, Kurian R, Jeejeebhoy K. Increased lipid peroxidation after lipid infusion as measured by breath pentane output. Am J Clin Nutr 1988; 48: 1394-1399

18. Lemoyne M, Van Gossum A, Kurian R, Jeejeebhoy K N. Plasma vitamin $\mathrm{E}$ and selenium and breath pentane in home parenteral nutrition patients. Am J Clin Nutr 1988; 48: 1310-1315

19. Siderova V S, Richelle M, Dubois D, Van Gossum A, Sultan F, Carpentier Y A. Intravenous (i.v.) $\alpha$-tocopherol supplementation in patients receiving long-term home parenteral nutrition (HPN). Clin Nutr 1995; 14: 47-48

20. Yeh S L, Chang K Y, Huang P C, Chen W J. Effects of n-3 and n-6 fatty acids on plasma eicosanoid and liver antioxidant enzymes in rats receiving total parenteral nutrition. Nutrition 1997; 13: $32-36$

21. Folch J, Lees M, Stanley G H S. A simple method for the isolation and purification of total lipids from animal tissues. J Biol Chem 1957; 226: 497-509

22. Butte W. Rapid method for the determination of fatty acid profiles from fats and oils using trimethylsulfonium hydroxide for transesterification. J Chromatogr 1983; 261: 142-145

23. Hess D, Keller H E, Oberlin B, Bonfanti R, Schüep W. Simultanous determination of retinol, tocopherols, carotenes and lycopene in plasma by means of high-performance liquid chromatography on reversed phase. Int J Vit Nutr Res 1991; 61: 232-238

24. Speitling A, Hüppe R, Kohlmeier M, Matiaske B, Stelte W, Thefeld W, Wetzel S. Methodenhandbuch der Verbundstudie Ernahrungserhebung und Risikofaktoren Analytik. VERA series, Vol. I, Niederkleen: Wissenschaftlicher Fachverlag Dr Fleck, 1992

25. Welz B, Schlemmer G, Mudakavi J R. Palladium nitrate - magnesium nitrate modifier for graphite furnance atomic absorption spectrometry. J Anal Atomic Spec 1988; 3: 93-97

26. Whitehead T P, Thorpe G H G, Maxwell S R J. Enhanced chemiluminescent assay for antioxidant capacity in biological fluids. Anal Chim Acta 1992; 266: 265-277

27. Linseisen J, Hoffmann J, Riedl J, Wolfram G. Effect of a single oral dose of antioxidant mixture (vitamin E, carotenoids) on the formation of cholesterol oxidation products after ex vivo LDL oxidation in humans. Eur J Med Res 1998; 3: 5-12

28. Lienhard S, Barlage S, Bolder U, Wilhelm-Heick B, Rothe G, Schmitz G, Jauch K-W. Effects of omega-3 fatty acids on natural killer cell activity and lymphocyte subpopulations in patients receiving total parenteral nutrition. Clin Nutr 1998; 17: 59

29. König W, Schönfeld W, Raulf M, Köller M, Knöller J, Scheffer J, Brom J. The neutrophil and leucotriens - role in health and diesease. Eicosanoids 1990; 3: 1-22

30. Rabl H, Khoschsorur G, Hauser H, Petek W, Esterbauer H. Diminished production of malondialdehyd after carotid artery surgery as a result of vitamin administration. Med Sci Res 1996; 24 $777-780$

31. Rabl H, Ratschek M, Khoschsorur G, Uranüs S, Tatzber F, Tillian H $\mathrm{M}$, Esterbauer $\mathrm{H}$. Limitation of intestinal reperfusion injury in the rat by vitamin treatment. Med Sci Res 1997; 25: 315-318

32. Janero D R. Therapeutic potential of vitamin E against myocardial ischemic reperfusion injury. Free Rad Biol Med 1991; 10: 315-324

33. Marubayashi S, Dohi K, Ochi K, Kawasaki T. Role of free radicals in ischemic rat liver cell injury: prevention of damage by $\alpha$-tocopherol administration. Surgery 1985; 99: 184-191

34. Steger P J K, Muhlebach S F. Lipid peroxidation in i.v. lipid emulsions in TPB bags: the influence of tocopherols. Nutrition 1998; 14: $179-185$

35. Bowry V W, Ingold K U, Stocker R. Vitamin E in human low density lipoprotein. when and how this antioxidant becomes a pro-oxidant. Biochem J 1992; 88: 341-344

36. Weber P, Bendich A, Machlin L J. Vitamin E and human health: rationale for determining recommended intake levels. Nutrition 1997 13: $450-460$

37. Pongracz G, Weiser H, Matzinger D. Tocopherols - antioxidants in nature. Fat Sci Technol 1995; 97: 90-104

38. Traber M G, Carpentier Y A, Kayden H J, Richelle M, Galeano N F, Deckelbaum R J. Alterations in plasma $\alpha$ - and $\gamma$-tocopherol concentrations in respose to intravenous infusion of lipid emulsions in humans. Metabolism 1993; 42: 701-709

39. Vandewoude M G, Vandewoude M F J, De Leeuw I H. Vitamin E status in patients on parenteral nutrition receiving Intralipid. JPEN 1986; 10: 303-305

40. Kelly F J, Sutton G L J. Plasma and red blood cell vitamin E status of patients on total parenteral nutrition. JPEN 1989; 13 : $510-515$

41. Biesalski $\mathrm{H} \mathrm{K}$, Böhles $\mathrm{H}$, Esterbauer $\mathrm{H}$ et al. Antioxidant vitamins in prevention. Dt Ärztebl 1995; 92: A1316-1321

42. Heseker H, Schneider R, Moch K J, Kohlmeier M, Kübler W. Vitaminversorgung Erwachsener in der Bundesrepublik Deutschland. VERA series, Vol. IV, Niederkleen: Wissenschaftlicher Fachverlag Dr Fleck, 1992

43. Furr H C, Clark R M. Intestinal absorption and tissue distribution of carotenoids. J Nutr Biochem 1997; 8: 364-377

44. Kohlmeier M, Thefeld W, Stelte W et al. Versorgung Erwachsener mit Mineralstoffen und Spurenelementen in der Bundesrepublik Deutschland. VERA series, Vol. V, Niederkleen: Wissenschaftlicher Fachverlag Dr Fleck, 1995

45. Rock C L. Carotenoids: biology and treatment. Pharmacol Ther 1997 75: $185-197$

46. Lissi E, Salim-Hanna M, Pascual C, del Castillo M D. Evaluation of total antioxidant potential (TRAP) and total antioxidant reactivity 
from luminol-enhanced chemiluminiscence measurements. Free Rad Biol Med 1995; 18: 153-158

47. Jialal I, Freeman D A, Grundy S M. Varying susceptibility of different low density lipoproteins to oxidative modification. Arterioscler Thromb 1991; 11: 482-488

48. Sevanian A, Hodis H N, Hwang J, McLeod L L, Peterson H.
Characterization of endothelial cell injury by cholesterol oxidation products found in oxidized LDL. J Lipid Res 1995; 36: 1971-1986

49. Linseisen J, Wilhelm M, Hoffmann J, Hailer S, Keller Ch, Wolfram G. Acute effects of LDL-apheresis on cholesterol oxidation products and antioxidants in plasma and lipoproteins of patients with familial hypercholesterolemia. Eur J Med Res 1999; 4: 433-441 